\title{
Comunicação
}

[Communication]

\section{Ocorrência de microsporídios em pequenos mamíferos silvestres no Estado de São Paulo}

[Occurrence of Microsporidia in small wildlife mammals in the state of São Paulo, Brazil]

\author{
A. Pereira ${ }^{1,2}$, R.S. Araújo ${ }^{1,3}$, S.E. Favorito ${ }^{2}$, P.B. Bertolla ${ }^{4}$, M.A. Lallo $^{2}$ \\ ${ }^{1}$ Hospital das Clínicas - FM-USP - São Paulo, SP \\ ${ }^{2}$ Universidade Bandeirante de São Paulo - São Paulo, SP \\ ${ }^{3}$ Faculdade de Saúde Pública - USP - São Paulo, SP \\ ${ }^{4}$ Faculdade de Medicina Veterinária e Zootecnia - USP - São Paulo, SP
}

\begin{abstract}
Microsporídios são parasitas intracelulares obrigatórios, que se encontram agrupados no filo Microspora. São considerados eucariontes, embora possuam características de organismos procariontes, tais como a ausência de mitocôndrias e peroxissomas. Estes microrganismos infectam uma variedade de tipos celulares de hospedeiros invertebrados e vertebrados, incluindo o homem (Franzen e Müller, 1999).
\end{abstract}

A transmissão da microsporidiose ocorre por meio da ingestão ou inalação dos esporos. Os microsporídios causam desde infecções intestinais a sistêmicas, sendo que os aspectos patogênicos da doença variam em função da espécie afetada e da competência da resposta imune do hospedeiro (Franzen e Müller, 1999). Várias espécies de microsporídios que infectam o homem têm sido confirmadas em animais domésticos, de laboratório e silvestres, incluindo roedores, porcos, cachorros, raposas, coelhos, macacos, gatos, burro, gado, caprinos, pássaros, répteis e anfíbios (Bornay-Llinares et al., 1998; Lallo e Bondan, 1999; Didier et al., 2000; Lores et al., 2002; Sulaiman et al., 2003). A possibilidade de que a microsporidiose seja uma doença zoonótica tem sido admitida (Didier et al., 1996; Dengjel et al., 2001).

Apesar de a veiculação hídrica ainda não ser bem conhecida, vários microsporídios têm sido encontrados em amostras de água de superfície (Avery e Undeen, 1987). Estudos recentes, envolvendo técnicas de biologia molecular, têm identificado a presença destes parasitas em esgotos, efluentes, água de superfície e também em águas profundas na França e nos Estados Unidos, reforçando o fato de que estes parasitas podem ser veiculados pela água (Sparfel et al., 1997; Dowd et al., 1998).

A microsporidiose já foi descrita em pequenos mamíferos silvestres, sugerindo que esses animais possam ser reservatórios de microsporídios, podendo constituir importantes fontes de infecção para o homem (Sulaiman et al., 2003). No Brasil, até o momento, não se tem registro de levantamentos sobre a infecção de pequenos animais silvestres por microsporídios.

Este trabalho teve como objetivo investigar a ocorrência de microsporídios em pequenos animais silvestres de uma região de desmatamento de mata nativa em São Paulo.

O estudo foi realizado nas áreas das planícies aluviais da Mata Atlântica da região sudoeste, no Estado de São Paulo, no local de implantação da barragem do rio Paraitinga e nas interligações do rio Tietê e do rio Biritiba-Mirim. Estas áreas, apesar de já serem influenciadas pela ação humana, ainda possuem uma diversidade muito grande de animais silvestres, principalmente de pequenos mamíferos. Algumas das dificuldades encontradas por pesquisadores, ao estudarem a ocorrência de parasitas em animais nestas regiões, são a escassez de inventários faunísticos

Recebido em 31 de março de 2008

Aceito em 31 de agosto de 2009

E-mail: adpee@uol.com.br 
e problemas relativos à taxonomia dos marsupiais e pequenos roedores de pequeno porte (Olio e Franco, 2004). Neste estudo, em paralelo, foi realizada a caracterização da fauna silvestre, facilitando, assim, a identificação dos animais utilizados nesta pesquisa.

Os animais foram apreendidos utilizando-se armadilhas de intercepção e queda (pit fall traps), eficientes na captura de vertebrados de pequeno porte, como anfíbios, répteis e pequenos mamíferos, e armadilhas de contenção viva (Shermann, Tomahawk), utilizadas para captura de pequenos e médios vertebrados terrestres e arborícolas. Os morcegos foram capturados com redes de neblina, abertas por seis horas, ao entardecer. Foram realizadas cinco campanhas de captura com duração de catorze dias cada, nos seguintes períodos: (1) antes do início dos trabalhos de desmatamento, (2) ao final do desmatamento, (3) no meio do período de enchimento, (4) imediatamente após a conclusão do enchimento e (5) transcorridos mais quatro meses.

Foram examinadas 47 amostras de fezes de diferentes populações de roedores, marsupiais, répteis, anfíbios, primatas e morcegos, colhidas nas armadilhas. As amostras foram mantidas em tubos com solução de dicromato de potássio a 2,5\%, na proporção de $3: 1$ e, em seguida, transportadas em condições adequadas para o laboratório.
Para a detecção de esporos de microsporídios, foram realizados esfregaços finos das amostras de fezes conservadas dos animais, após serem homogeneizadas e coradas pela técnica de GramCromotrope (Moura et al., 1996), a mesma adotada na rotina para a identificação de esporos desses parasitas e diagnóstico da microsporidiose no laboratório de parasitologia do Hospital das Clínicas da Faculdade de Medicina da Universidade de São Paulo (Gonçalves et al., 2006). Pesquisadores brasileiros que trabalham com infecção experimental também utilizam essa coloração para detecção e demonstração dos esporos de microsporídios (Lallo, 1998; Lallo et al., 2002; Lallo e Bondan, 2005; Pereira et al., 2005). Esta, diferente da coloração de Gram, permite diferenciar os esporos de microsporídios de bactérias e fungos. Algumas amostras foram confirmadas com a técnica tricrômica modificada (Weber et al., 1992). As lâminas coradas foram observadas em microscópio de luz no aumento de 1000x.

Foram encontrados esporos de microsporídios em fezes de seis $(27,3 \%)$ pequenos roedores (Akodon sp., Oligoryzomys sp. e Coendou), três (33,3 \%) marsupiais (Marmosa sp. e Didelphis aurita) e três $(60 \%)$ morcegos do gênero Diphylla. Não foram encontrados esporos nas amostras dos anfíbios $(\mathrm{n}=5)$, dos macacos $(\mathrm{n}=$ 5) e na amostra da lontra $(n=1)$.

Tabela 1. Amostras de fezes analisadas para detecção de esporos de microsporídios em pequenos animais $\underline{\text { silvestres em área de desmatamento em São Paulo }}$

\begin{tabular}{cccc}
\hline Grupo de animais & $\begin{array}{c}\mathrm{N}^{\mathrm{o}} \text { de amostras } \\
\text { analisadas }\end{array}$ & $\begin{array}{c}\mathrm{N}^{\circ} \text { de animais } \\
\text { positivos }\end{array}$ & $\begin{array}{c}\text { Porcentagem } \\
(\%)\end{array}$ \\
\hline Pequenos roedores* & 22 & 6 & 27,27 \\
Marsupiais** & 9 & 3 & 33,3 \\
Macacos (Callithrix) & 5 & 0 & 0 \\
Morcegos (Diphylla) & 5 & 3 & 60,0 \\
Sapos (Bufo) & 5 & 0 & 0 \\
Lontra & 1 & 0 & 0 \\
Total & 47 & 12 & 25,53 \\
\hline
\end{tabular}

*Akodon sp., A. montensis, Oligoryzomys nigripes, Oryzomys russatus, Juliomys pictipes, Thaptomys, Coendou, Sciurus sp. ** Marmosa sp., M. americana, Didelphis aurita, Gracilinamus.

À microscopia de luz, no aumento de 1000x (imersão), os esporos de microsporídios mostravam-se como estrutura ovoide, ligeiramente curvada, com medidas que variavam de 2,2 a $3,2 \mu \mathrm{m}$ de comprimento por 1,0 a 1,6 $\mu \mathrm{m}$ de largura. Foram observados, em todas as amostras positivas, muitos esporos com um halo transparente na região equatorial, característico dos esporos de microsporídios. 
Microsporídios são encontrados em diferentes mamíferos, incluindo o homem. No Brasil, esses parasitas foram identificados principalmente em indivíduos imunossuprimidos (Brasil et al., 1999; Gonçalves et al., 2006). O presente estudo é o único, até o momento, que demonstra a presença desses parasitas em pequenos mamíferos silvestres no país.

Animais podem servir como fonte de infecção por microsporídios para humanos. A identificação de esporos desses parasitas pode reduzir e prevenir a exposição do homem. Isso se aplica principalmente para pessoas que possuem um maior risco de adquirir microsporidiose, como no caso de imunocomprometidos e crianças desnutridas (Lobo et al., 2003).

No presente trabalho, esporos de microsporídios foram identificados em pequenos mamíferos silvestres, no entanto não se sabe se a presença dos esporos nas amostras de fezes é resultado de uma infecção ativa ou uma simples passagem dos esporos através do trato gastrointestinal desses animais. Embora essa questão não tenha sido esclarecida, os resultados desse trabalho sugerem que a exposição a excrementos desses animais pode ser um fator potencial para causa de infecções em humanos.

Acredita-se que os resultados desse estudo sejam utilizados para o estabelecimento de possíveis rotas de transmissão e que contribuam para o conhecimento da epidemiologia destes agentes infecciosos.

Palavras-chave: microsporídia, pequenos mamíferos silvestres

\section{ABSTRACT}

This study describes infection of microsporidia in the natural fauna of small wildlife animals in an area of deforestation for a water reservoir construction in the state of São Paulo, Brazil. It was focused on marsupials, small rodents, frogs, primates, bats, and others mammals taken from this area to access whether they may represent environmental sources of these zoonoses. From all captured animals, microsporidia spores were observed in the feces of bats, small rodents, and marsupials. This study emphasises the importance of wild animals, particularly small mammals as potential sources of microsporidia to human and animal populations from deforested areas.

Keywords: microsporidia, small wildlife mammals

\section{REFERÊNCIAS BIBLIOGRÁFICAS}

AVERY, S.W.; UNDEEN, A.H. The isolation of microsporidia and other pathogens from concentrated ditch water. J. Am. Mosq. Control Assoc., v.3, p.54-58, 1987.

BORNAY-LLINARES, F.J.; SILVA, A.J.; MOURA, H. et al. Immunologic, microscopic, and molecular evidence of Encephalitozoon intestinalis (Septata intestinalis) infection in mammals other than humans. J. Infect. Dis., v.178, p.820-826, 1998.

BRASIL, P.; LIMA, D.B.; PAIVA, D.D. et al. Emerging and opportunistic intestinal parasites in HIV-infected patients with chronic diarrhea in Rio de Janeiro, Brazil. J. Eukaryot Microbiol., v.46, p.40S-41S, 1999.
DENGJEL, B.; ZAHLER, M.; HERMANNS, W. et al. Zoonotic potential of Enterocytozoon bieneusi. J. Clin. Microbiol., v.39, p.4495-4499, 2001.

DIDIER, E.S.; DIDIER, P.J.; SNOWDEN, K.F. et al. Microsporidiosis in mammals. Microbes Infect., v.2, p.709-720, 2000.

DIDIER, E.S.; VISVESVARA, G.S.; BAKER, M.D. et al. A microsporidian isolated from an AIDS patient corresponds to Encephalitozoon cuniculi III, originally isolated from domestic dogs. J. Clin. Microbiol., v.34, p.2835-2837, 1996.

DOWD, S.E.; GERBA, C.P.; PEPPER, I.L. Confirmation of the human-pathogenic microsporidia Enterocytozoon bieneusi, Encephalitozoon istestinalis, and Vittaforma corneae in water. Appl. Environ. Microbiol., v.64, p.3332-3335, 1998. 
FRAZEN, C.; MÜLLER, A. Molecular techniques for detection, species differentiation, and phylogenetic analysis of microsporidia. Clin. Microbiol. Rev., v.12, p.243-285, 1999.

GONÇALVES, E.M.N.; UEMURA, I.H.; ORBAN, M. et al. Microsporidiosis in a brazilian university hospital. case report. Rev. Inst. Med. Trop. São Paulo, v.48, p.351-352, 2006.

LALLO, M.A. Estudo da infecção experimental pelo Encephalitozoon cuniculi em camundongos Balb-c tratados com ciclofosfamida ou ciclosporina. 1998. 175f. Tese (Doutorado) Faculdade de Medicina Veterinária e Zootecnia, Universidade de São Paulo, São Paulo.

LALLO, M.A.; BONDAN, E.F. Encefalitozoonose canina: revisão. Clin. Vet., n.19, p.26-30, 1999.

LALLO, M.A.; BONDAN, E.F. Experimental meningoencephalomyelitis by Encephalitozoon cuniculi in cyclophosphamideimmunosuppressed mice. Arq Neuropsiq., v.63, p.246-251, 2005

LALLO, M.A.; SANTOS, M.J.; BONDAN, E.F. Infecção experimental pelo Encephalitozoon cuniculi em camundongos imunossuprimidos com dexametasona. Rev. Saúde Publica, v.36, p.621-626, 2002.

LOBO, M.L.; TELES, A.; CUNHA, M.B. et al. Microsporidia detection in stools from pets and animals from the zoo in Portugal: a preliminary study. J. Eukaryot Microbiol., v.50, p.581-582, 2003.

LORES, B.; AGUILA, C.; ARIAS, C. Enterocytozoon bieneusi (microsporidia) in feacal samples from domestic animals from Galicia, Spain. Mem. Inst. Oswaldo Cruz, v.97, p.941-945, 2002.
MOURA, H.; SILVA, J.L.; SODRÉ, F.C. et al. Gram-Chromotrope: a new technique that enhances detection of microsporidial spores in clinical samples. J. Eukaryot Microbiol., v.43, p.94-95, 1996.

OLIO, A.J.D.; FRANCO, R.M.B. Ocorrência de Cryptosporidium spp. em pequenos mamíferos silvestres de três áreas serranas do Sudeste brasileiro. Arq. Bras. Med. Vet. Zootec., v.56, p.25-31, 2004.

PEREIRA, A.; LALLO, M.A.; ARAÚJO, R.S. et al. Diagnóstico da microsporidiose por técnicas de coloração em exames histopatológicos. Rev. Inst. Adolfo Lutz, v.64, p.229, 2005. (Resumo).

SPARFEL, J.M.; SARFATI, C.; LIGUORI, O. et al. Detection of microsporidia and identification of Enterocytozoon bieneusi in surface water by filtration followed by specific PCR. J. Eukaryot Microbiol., v.44, p.78S, 1997. (Resumo).

SULAIMAN, I.M.; FAYER, R.; LAL, A.A. et al. Molecular characterization of microsporidia indicates that wild mammals harbor host-adapted Enterocytozoon spp. as well as humanpathogenic Enterocytozoon bieneusi. Appl. Environ. Microbiol., v.69, p.4495-4501, 2003.

WEBER, R.; BRYAN, E.R.; OWEN, R.L. et al. Improved light-microscopical detection of microsporidia spores in stool and duodenal aspirates. N. Engl. J. Med., v.326, p.161-166, 1992. 